\title{
Adaptação e validação da Escala de Automonitoria para o português do Brasil
}

\author{
Marcia Cristina Monteiro', Adriana Benevides Soares \\ Universidade Salgado de Oliveira, Niterói-RJ, Brasil
}

\section{RESUMO}

O presente estudo teve como objetivo a adaptação e validação da Escala de Automonitoria. A amostra contou com 200 estudantes universitários, dos quais 50\% eram advindos de Instituições de Ensino Superior (IES) particulares e 50\% de IES públicas. A coleta dos dados ocorreu nesses locais. A análise fatorial exploratória indicou a extração de dois fatores e a exclusão de oito itens. Apesar do índice de confiabilidade da escala, os resultados não podem ser generalizados para um público mais amplo do que aquele formado por estudantes universitários. Além disso, alguns itens apresentaram valores pouco expressivos de correlação. Assim, uma pesquisa futura poderia investigar a estrutura obtida e se esta permanece sem alterações significativas quando replicada em outras amostras.

Palavras-chave: automonitoria; autorregulação; interação.

\section{ABSTRACT - Adaptation and validation of the Self-monitoring Scale for Brazilian Portuguese}

This study aimed to adapt and validate the Self-Monitoring Scale. The sample consisted of 200 college students, 50\% coming from private Higher Education Institutions and 50\% from public ones. Data collection occurred in these places. The instrument used was a revised version of a Self-Monitoring Scale. The exploratory factor analysis indicated the extraction of two factors and the exclusion of eight items. Despite the scale's reliability, the results cannot be generalized to a wider audience than the one formed by college students. In addition, some items showed few significant values of correlation. Thus, future research could investigate the structure obtained for this range and determine if no significant changes when replicated in other samples.

Keywords: self-monitoring; self-regulation; interaction.

\section{RESUMEN - Adaptación y validación de la escala Automonitoramento para el portugués de Brasil}

Este estudio tuvo como objetivo la adaptación y validación de la Escala Automonitoramento. La muestra estuvo constituida por 200 estudiantes universitarios, de los cuales un 50\% era procedente de Instituciones de Educación Superior (IES) públicas y un 50\% de IES privadas. Los datos fueron recolectados en estos lugares. El análisis factorial exploratorio indicó la extracción de dos factores y la exclusión de ocho artículos. A pesar del índice de fiabilidad de la escala, los resultados no pueden generalizarse a un público más amplio que el formado por los estudiantes universitarios. Además, algunos artículos mostraron pequeños valores significativos de correlación. Por lo tanto, la investigación futura podría investigar la estructura obtenida para esta gama y se mantiene sin cambios significativos cuando replicado en otras muestras.

Palabras clave: automonitoramento; autorregulación; interacción.

Os desafios encontrados na rotina universitária podem ser considerados de difícil manejo para muitos estudantes. Atividades como iniciar novas amizades, lidar com diferentes componentes curriculares e de forma menos sistematizada, conhecer diferentes procedimentos de avaliação, habituar-se à didática docente, falar em público e lidar com grupos são alguns exemplos de situações a serem administradas pelos alunos no Ensino Superior (Soares, Poubel, \& Mello, 2009) e que podem intervir na adaptação acadêmica. As questões que envolvem a integração do aluno ao ensino universitário tem sido uma crescente preocupação dos pesquisadores, tanto no cenário nacional como no internacional. Segundo Almeida e Castro (2016), o tema vem sendo devidamente abordado pela ótica da expansão e democratização do acesso à universidade. No entanto, segundo os autores, pesquisas sobre a permanência do aluno na universidade e a formação com qualidade deveriam receber maior enfâse, priorizando aspectos de cunho acadêmico, pessoal, vocacional e social, além de questões acadêmicas.

No que diz respeito às questões sociais de adaptação do universitário, a habilidade de adequar a forma de expressar comportamentos nas relações com pares, professores, gestores e funcionários muito facilitaria o estudante, 
pois este teria melhores condições de administrar impasses gerados nas relações interpessoais (Bardagi \& Hutz, 2012; Kyalo \& Chumba, 2011; Pérez, Santiviago, Aguilar, DaRe, \& Rubio, 2014). Segundo Polanco et al. (2014), para que houvesse uma melhor integração do aluno este deveria ser responsável pelo aproveitamento das oportunidades oferecidas no contexto universitário, tanto para a formação profissional quanto para o desenvolvimento psicossocial. Estudantes que desde o início do curso se integram à vida universitária focam nos objetivos que pretendem alcançar na futura profissão, aprimoram seus relacionamentos e tendem a maiores chances de crescimento em termos cognitivos e afetivos, se comparados àqueles com dificuldades na transição de modalidade de ensino (Teixeira, Dias, Wottrich, \& Oliveira, 2008).

Nesse sentido, a universidade pode exigir do estudante procedimentos mais maduros nas relações interpessoais com a comunidade acadêmica. $\mathrm{O}$ desenvolvimento da habilidade de automonitoria muito facilitaria o processo de adaptação do aluno. Ickes, Holloway, Stinson e Hoodenpyle (2006) sinalizam que a automonitoria mobiliza um grande esforço cognitivo e emocional no intuito da pessoa administrar o desempenho social, visando o sucesso nas relações com o grupo ou com o outro. Os esforços envolvem mudanças e ajustamentos das ações à dinâmica das situações, visando ao êxito nos relacionamentos e tentando alcançar os objetivos pretendidos com a interação.

O conceito de automonitoria (Snyder, 1974; 1987) refere-se às diferenças individuais na habilidade de regular a expressão de comportamentos e a aparência pública, considerando os aspectos externos para obtenção de pistas relevantes para o desempenho. Leone (2006) sinaliza que o construto se expandiu ao longo de quatro décadas de estudos, apresentando relação teórica e empírica com o autoconceito e com as relações interpessoais.

Fuglestad e Snyder (2010) apontam que pessoas que têm escores altos em automonitoria são claramente sensíveis ao contexto situacional, desejosos e capazes de modificar a expressão de comportamento para se ajustarem à situação ou ao papel. Por outro lado, as que apresentam baixo escore são menos responsivas ao contexto social, agindo tipicamente de maneira congruente com suas disposições internas. De acordo com Leone (2006), as pessoas com elevada automonitoria agem pelas contingências situacionais, conforme os papéis desempenhados, e têm mais chances de se engajarem em novos relacionamentos do que os com baixos escores, pois conseguem utilizar diferentes processos comportamentais para iniciarem suas relações. Já os indivíduos com baixa automonitoria tendem a se orientar por aspectos disposicionais, sendo condescendentes diante de situações adversas.

A universidade é um espaço no qual o estudante terá que aprender a manejar novas relações, expressando comportamentos que promovam e facilitem o processo de aprendizagem e os vínculos a serem construídos com a comunidade universitária. Nesse sentido, é fato que as pessoas diferem na forma como controlam e expressam ideias e sentimentos. Há aquelas que são mais sensíveis diante das pistas sociais e interpessoais, regulando a autoapresentação e adequando-se às situações do contexto. Por outro lado, há as que não se engajam de forma tão concernente no controle das diferentes formas de autoexpressão, sendo mais atentas às suas questões internas, aos seus sentimentos, às suas atitudes e emoções (Barreiros, 2011; Gangestad \& Snyder, 2000), sinalizando a importância de se mensurar a automonitoria como variável relevante no processo de adaptação do aluno à universidade.

A primeira escala de automonitoria (Snyder, 1974) foi constituída de 25 itens dicotômicos $(\alpha=0,83)$ e validada em grupos de estudantes universitários, atores e pacientes psiquiátricos. O autor concebeu a escala para medir um construto unidimensional. Snyder e Gangestad (1986) revisaram a escala de 25 itens, excluindo 7 deles $(\alpha=0,70)$. Segundo os autores, o objetivo foi aumentar a confiabilidade da escala, mantendo a estabilidade intrínseca. A análise da estrutura latente indicou que alguns itens não diferenciavam adequadamente alto e baixo automonitores. A revisão indicou ainda que o primeiro fator não rotacionado foi responsável por $62 \%$ da variância comum, comparado aos 51\% obtidos na escala original de 25 itens.

O modelo proposto por Lennox e Wolfe (1984) é composto por 13 itens $(\alpha=0,75)$ e denominado Escala de Automonitoria Revisada. Os autores consideraram as diferenças individuais na sensibilidade de expressar o comportamento do outro e na habilidade de modificar a autoapresentação. A escala é constituída de duas subescalas: habilidade de modificar a autoapresentação $(\alpha=0,77)$ e sensibilidade à expressão do comportamento do outro $(\alpha=0,70)$. Recomenda-se que os pesquisadores utilizem a resposta ao instrumento do tipo Likert de seis pontos, devido ao fato dos estudos realizados terem mostrado uma relativa estabilidade de correlação nas matrizes.

Barreiros (2011), em pesquisa sobre a relação entre automonitoria e prossociabilidade, e considerando o número de fatores subjacentes e que a estrutura de relações com os itens da Escala de Automonitoria Revisada de 18 itens não apresenta consenso na literatura quanto ao número de fatores - um de Gangestad e Snyder (2000); dois de Briggs e Cheek (1988); e três de Briggs, Cheek e Buss (1980) —, realizou análise fatorial confirmatória adotando os três modelos citados. Para tal, o pesquisador utilizou a Escala de Automonitoria de 18 itens adaptada por Amorim (2001) para o português de Portugal $(\alpha=0,76)$. A amostra contou com 100 estudantes universitários de ambos os sexos - $49 \%$ homens e $51 \%$ mulheres - e idades entre 17 e 34 anos $-(M)=21,84$ e $(D P)=2,98$. $\mathrm{O}$ resultado apontou que apenas o modelo de dois fatores apresentou índices de qualidade de ajustamento satisfatórios - comparative fit index $(\mathrm{CFI})=0,87$ e root mean square error of approximation (RMSEA) $=0,06$. 
Perante essa divergência de indicadores de qualidade de ajustamento, a escala foi submetida à análise de componentes principais, o que resultou na extração de sete fatores e na apresentação de uma confiabilidade de 0,75 (Barreiros, 2011), sendo que o primeiro fator explicou $21 \%$ da variância. Para o autor, esses dados afirmaram a natureza psicométrica instável da Escala de Automonitoria ao nível da estrutura fatorial sinalizada pela literatura (Leone, 2006; Wilmot, 2015). Evidências apontam para o fato de que tanto a escala de 25 itens como a de 18 são multidimensionais. O modelo revisado de 18 itens foi reconhecido como aquele que possui as melhores qualidades psicométricas (Briggs \& Cheek, 1988), tornando-se o mais usado (Barreiros, 2011; Leone, 2006). Wilmot (2015) ressalta que a escala foi traduzida para o alemão (Nowack \& Kammer, 1987), queniano (Kodero, 1991), português de Portugal (Amorim, 2001; Neto, 1993), espanhol (Avia, Sánchez-Bernardos, Sanz, Carrillo, \& Rojo, 1998) e hebraico (Bachner-Melman et al., 2009), obtendo dois fatores ortogonais na escala de 18 itens.

Pesquisas empíricas nas duas últimas décadas utilizaram a versão de 18 itens da Escala de Automonitoria, procurando relacionar o comportamento de alto e baixo automonitores com diferentes variáveis (Oyamot, Fuglestad, \& Snyder, 2010; Sasovova, 2006; Soibel, Fong, Mullin, Jenkins, \& Mars, 2012). No entanto, apenas um estudo na literatura foi encontrado, ao longo de anos de pesquisas, envolvendo a temática de adaptação à universidade.

Guarino, Michael e Hocevar (1998) realizaram um estudo com 380 estudantes de Psicologia utilizando a Escala de Automonitoria de 18 itens. O estudo apontou que os alunos que apresentam alto escore em automonitoria são capazes de ajustar seu comportamento e promover integração social com seus pares. A pesquisa citada mostrou ainda que os participantes do sexo masculino classificados com altos escores em automonitoria apresentaram bom manejo das relações interpessoais. Por outro lado, indicou também que os participantes classificados com baixos escores em automonitoria apresentaram melhor engajamento acadêmico, quando comparados aos do outro grupo.

Considera-se que para um processo de adaptação acadêmica de sucesso, o estudante também precise construir novas relações e que, para tal, é necessário saber controlar a maneira de se apresentar e expressar seu comportamento. Dessa forma, e considerando que não foram encontrados outros instrumentos de automonitoria validados para o português e para a realidade brasileira, o presente estudo teve como objetivo a adaptação e validação da Escala de Automonitoria de Snyder e Gangestad (1986).

\section{Método}

\section{Participantes}

Participaram do estudo 200 estudantes universitários, dos quais 50\% (100) são advindos de Instituições de Ensino Superior (IES) particulares e 50\% (100) de IES públicas, selecionados por conveniência. Desses, 38\% dos voluntários eram do sexo masculino e $62 \%$ do sexo feminino, sendo que 41 (20,5\%) estudantes das IES públicas responderam aos instrumentos online. Concernente aos participantes das IES particulares, $22 \%$ eram do sexo masculino e $78 \%$ do feminino. Tantos os alunos das instituições públicas quanto os das particulares eram de diferentes cursos de graduação. Em termos de período, 24,0\% eram alunos do primeiro semestre; $8,5 \%$ do segundo; $18,5 \%$ do terceiro; $36,0 \%$ do quarto ao oitavo; e $13,0 \%$ do nono ao décimo. A idade variou entre 18 e 56 anos, com média de 25,4 anos $(D P=8,03)$. Em relação à classe econômica, constatou-se que, entre os participantes das IES particulares, $10 \%$ eram pertencentes à classe $\mathrm{A} ; 13 \%$ à $\mathrm{B} 1 ; 22 \%$ à $\mathrm{B} 2 ; 25 \%$ à $\mathrm{C} 1$; $19 \%$ à C2; e 2\% à DE. Entre os estudantes das IES públicas, por sua vez, $9 \%$ são da classe A, $18 \%$ da B1, 43\% da B2, $16 \%$ da C1, 5\% da C2 e 3\% da DE, segundo o Critério de Classificação Econômica Brasil da Associação Brasileira de Empresas de Pesquisa (ABEP) (2015).

\section{Instrumento}

A Escala de Automonitoria (Snyder \& Gangestad, 1986) é composta por 18 itens afirmativos, com opção de marcação do tipo verdadeiro ou falso $(\alpha=0,70)$. Para os autores, a escala apresenta três fatores: ação $(\alpha=0,78)$, extroversão $(\alpha=0,68)$ e direcionado para outro $(\alpha=0,39)$. Em estudo realizado por Briggs e Cheek (1988), os autores sustentam a extração de dois fatores: fluência social $(\alpha=0,86)$ e direcionamento para outro $(\alpha=0,39)$.

\section{Procedimentos}

A tradução e validação do instrumento envolveram seis etapas. Inicialmente, a tradução foi realizada por dois voluntários da área de Psicologia com fluência no inglês e no português. Posteriormente, dois nativos e fluentes nesses dois idiomas procederam à tradução do instrumento do português para o inglês (back-translation). Ajustes linguísticos e semânticos ainda necessários foram realizados por dois professores licenciados em português/inglês. A primeira versão do instrumento foi aplicada de forma piloto a seis alunos voluntários, sendo três do Ensino Médio e três do Ensino Superior. Os participantes responderam ao instrumento e solicitou-se que sugerissem possíveis ajustes.

O instrumento foi submetido a cinco juízes avaliadores - dois pós-doutores, dois doutores e um mestre em Psicologia. A validação do conteúdo ocorreu devido ao julgamento desses juízes, e os ajustes propostos pelos voluntários foram realizados, considerando o cuidado quanto às equivalências semânticas, idiomáticas e conceituais. Finalmente, o instrumento foi aplicado em uma amostra de 200 estudantes universitários. Todos os participantes responderam ao Questionário Sociodemográfico e assinaram o Termo de Consentimento Livre e Esclarecido (TCLE). O estudo foi aprovado pelo Comitê de Ética em Pesquisa da Universidade. 


\section{Análise de dados}

A análise psicométrica do instrumento foi realizada por meio de análise fatorial exploratória (AFE), utilizando o programa Factor 10.3 (Lorenzo-Seva \& Ferrando, 2006), adequado para dados categóricos (dicotômicos). Foi realizada análise de correlação policórica (Olsson, 1979a; 1979b) da variância/covariância, e para os casos de missings foi utilizada a imputação múltipla (Lorenzo-Seva \& Van Ginkel, 2015). Empregou-se a análise paralela com permutação com o banco de dados (Timmerman \& Lorenzo-Seva, 2011) para determinar o número de dimensões. A rotação oblíqua Promin foi utilizada para ativar a simplicidade dos fatores e a Varimax para confirmar a ortogonalidade deles. Finalmente, a extração de fatores deu-se por intermédio do minimum rank factor analysis (MRFA), de Ten Berge e Kiers (1991).

\section{Resultados}

A fatorialidade da matriz de dados foi confirmada pelo indicador Kaiser-Meyer-Olkin measure of sampling adequacy (KMO). O teste de esferacidade de Bartlett foi estimado em 298,0, o KMO em 0,73898 e graus de liberdade (df) em $45(p=0,000010)$. A análise fatorial exploratória utilizou a variância explicada por meio da retenção de fatores pelos autovalores (eigenvalues), conforme apresentado na Tabela 1.

Além disso, a extração de fatores por componentes principais indicou a retirada de oito itens $-1,2,3,8,9$, 10,11 e 15 - pelo fato de apresentarem carga fatorial (loadings) muito baixa - variação entre -1 a +1 sendo média 0 - e por não terem correlação com nenhum dos fatores na matriz de dados rotacionados — acima de 0,30. Outro aspecto levado em consideração para essa extração foram as comunalidades das variáveis. A análise paralela também indicou a manutenção de dois fatores.
A rotação dos dados na análise fatorial foi obtida por meio do método Varimax, indicando a ortogonalidade dos fatores, e apresentada na Tabela 2. As variáveis 12, 17 e 18 correlacionaram com o fator um (F1) e com o fator dois (F2). A variável quatro correlacionou negativamente com F2, apesar de não ter sido muito expressivo. As demais variáveis apresentaram cargas fatoriais consideradas fracas. A análise paralela também indicou a manutenção de dois fatores (F1=44,2 e F2=14,9), uma dimensão e a permanência de 10 itens na escala.

Por fim, a Tabela 3 mostra a matriz de correlação policórica. Os dados obtidos apontam para a discriminação de fatores que poderiam ser denominados de:

- F1 - Reações externalizantes: referem-se aos comportamentos apresentados pela pessoa para lidar com as demandas interativas do seu ambiente, por serem expressos predominantemente de forma aberta; e

Tabela 1

Variância explicada por meio dos eigenvalues

\begin{tabular}{lccc}
\hline Variável & Eigenvalues & $\begin{array}{c}\text { Proporção } \\
\text { da variância }\end{array}$ & $\begin{array}{c}\text { Proporção } \\
\text { acumulada } \\
\text { da variância }\end{array}$ \\
\hline 1 & 4,27338 & 0,42734 & 0,42734 \\
2 & 1,45969 & 0,14597 & 0,57331 \\
3 & 1,04160 & 0,10416 & \\
4 & 0,89990 & 0,08999 & \\
5 & 0,62355 & 0,06236 & \\
6 & 0,56299 & 0,05630 & \\
7 & 0,50947 & 0,05095 & \\
8 & 0,42598 & 0,04260 & \\
9 & 0,16092 & 0,1609 & \\
10 & 0,04254 & 0,00425 & \\
\hline
\end{tabular}

Tabela 2

Matriz de dados rotacionados por item da Escala de Automonitoria utilizando a Varimax

\begin{tabular}{lcc}
\hline Variável & F1 & F2 \\
\hline 4. Faço discursos de improviso até mesmo sobre temas que não tenho muitas informações. & 0,363 & $-0,454$ \\
5. Acho que faço um verdadeiro espetáculo para impressionar ou entreter os outros. & 0,370 & $-0,367$ \\
6. Provavelmente eu seria um bom ator. & 0,608 & $-0,291$ \\
7. Em um grupo de pessoas, raramente sou o centro das atenções. & 0,074 \\
12. Em diferentes situações com diferentes pessoas, frequentemente ajo de forma diferente & 0,640 \\
do que costumo ser. & 0,580 & $-0,391$ \\
13. Nunca fui bom em charadas ou improvisos. & $-0,080$ & 0,432 \\
14. Tenho problema em mudar meu comportamento para adequá-lo a diferentes pessoas e & 0,008 & 0,742 \\
situações. & 0,166 & 0,728 \\
16. Em uma festa deixo os outros levarem à frente piadas e histórias. & 1,017 \\
17. Sinto-me desconfortável em público e não me saio tão bem como poderia. & 0,310 \\
18. Posso enganar as pessoas sendo amigável, quando, na realidade, não gosto delas. & 0,960 & 0,233 \\
\hline
\end{tabular}

Nota: F1=fator 1; F2=fator 2. 
Tabela 3

Correlação e covariância da matriz de dados: correlação Policórica

\begin{tabular}{lccccccccc}
\hline & 4 & 5 & 6 & 7 & 12 & 13 & 14 & 16 & 17 \\
\hline 4 & 1,000 & & & & & & & & \\
5 & 0,428 & 1,000 & & & & & & & \\
6 & 0,272 & 0,506 & 1,000 & & & & & & \\
7 & $-0,435$ & $-0,432$ & $-0,210$ & 1,000 & & & & & \\
12 & 0,512 & 0,535 & 0,845 & 0,214 & 1,000 & & & & \\
13 & $-0,298$ & $-0,133$ & $-0,378$ & 0,177 & $-0,321$ & 1,000 & & & \\
14 & $-0,381$ & $-0,279$ & 0,310 & 0,366 & $-0,510$ & 0,423 & 1,000 & & \\
16 & 0,321 & $-0,294$ & $-0,381$ & 0,408 & $-0,290$ & 0,271 & 0,380 & 1,000 & \\
17 & 0,486 & 0,312 & 0,471 & $-0,223$ & 0,411 & $-0,197$ & $-0,173$ & 0,060 & 1,000 \\
18 & 0,404 & 0,428 & 0,479 & $-0,090$ & 0,512 & $-0,192$ & $-0,308$ & $-0,074$ & 0,762 \\
\hline
\end{tabular}

Nota: $0,001<p \leq 0,05$.

- $\quad$ F2 - Reações internalizantes: expressam-se por comportamentos que administram as contingências do contexto, principalmente de forma encoberta.

A estimativa da proporção da variância comum obtida para F1 foi de 0,366 e para F2, de 0,296, sendo a confiabilidade por fator (Mislevy \& Bock, 1990) de 0,921 (F1) e 0,836 (F2) da matriz rotacionada. É interessante lembrar que, na escala original de 18 itens - em inglês —, o coeficiente de alfa de Cronbach foi de 0,70 (Snyder \& Gangestad, 1986). Os casos de dados faltantes somam menos de $1 \%$ dos itens em branco dos 18 analisados, não apresentando um padrão de ocorrência. A estimativa dos dados indicou 2 fatores e a extração por componentes principais mostrou que os 2 primeiros fatores explicaram $56 \%$ da variância dos dados.

\section{Discussão}

O acesso e a permanência do estudante na universidade despertam preocupações sobre a qualidade da formação e dos aspectos facilitadores, assim como aqueles considerados de difícil manejo pelo aluno. Controlar a maneira de se autoapresentar é uma habilidade que, supostamente, auxilia as pessoas em suas relações interpessoais, e facilitaria ao estudante a consolidação de novas amizades e o convívio com professores, gestores e pessoal administrativo. A automonitoria (Barreiros, 2011; Leone, 2006; Gangestad \& Snyder, 1974; 1987; 2000) mostra-se como um aspecto importante e facilitador tanto na construção de novos vínculos como na consolidação daqueles já existentes. Ao longo das décadas, as pesquisas realizadas sobre automonitoria agregaram novos conhecimentos, inclusive no campo dos relacionamentos (Barreiros, 2011; Guarino et al., 1998; Oyamot et al., 2010; Sasovova, 2006). Como sinalizado, as relações interpessoais são importantes no contexto acadêmico e no sucesso profissional e pessoal (Bardagi \& Hutz, 2012; Kyalo \& Chumba,
2011; Pérez et al., 2014; Teixeira et al., 2008). Assim, a automonitoria parece colaborar na habilidade do estudante em enfrentar diferentes desafios que lhe são apresentados ao gerir novos vínculos, podendo promover a permanência do aluno e seu engajamento com qualidade à universidade.

Os resultados obtidos sugerem que os estudantes lidam com as situações levando em consideração tanto aspectos externos, percebidos nas vivências do cotidiano da universidade, como internos, relevantes para adequarem-se ao novo contexto e às novas amizades. As respostas obtidas em itens da escala podem indicar que, para a amostra de estudantes, ter habilidade de automonitoria estaria especificamente relacionado à condição de camuflar comportamentos no intuito de agradar ao outro. Consequentemente, é provável que compreendam que monitorar a expressão de ações consideradas menos apropriadas é uma forma de gerir melhor as situações de difícil manejo que possam ocorrer no grupo, buscando estratégias que favoreçam as relações interpessoais. Esses resultados apontam para o fato de que as pessoas que regulam o comportamento para as demandas externas vinculadas às diferentes situações vivenciadas preocupam-se com a imagem projetada no contexto, ajustando-se de forma mais apropriada à circunstância imposta (Smidt \& DeBono, 2011). Por outro lado, pode-se inferir que alunos que priorizam os aspectos internos apresentam maior dificuldade em administrar a expressão de comportamentos.

A Escala de Automonitoria de Gangestad e Snyder (2000) enfatiza três fatores que, segundo os autores, foram melhor expressos na versão revisada de 18 itens, não corroborado pelos dados desta pesquisa. No entanto, como já mencionado, não existe um consenso em relação ao número de fatores, apresentando de um a quatro. A estimativa de dois fatores é sustentada pela literatura sobre a escala (Briggs et al., 1980; Briggs \& 
Cheek, 1988; Wilmot, DeYoung, Stillwell, \& Kosinski, 2015), cujos fatores são denominados fluência social e direcionamento para outro.

O desenvolvimento do estudo transcultural de adaptação e validação da Escala de Automonitoria resultou em um instrumento em português do Brasil cuja estrutura alcançada manteve dez itens. Estudos desse tipo podem ser considerados substanciais, pois tentam contribuir com ferramentas aplicáveis em diferentes áreas da Psicologia.

Como apontado, a adaptação acadêmica é um fenômeno que envolve diferentes fatores que precisam ser melhor compreendidos, relacionados e mensurados. O estudo indicou que o fator externalizante (F1) poderia proporcionar expressão de comportamentos facilitadores na construção de novas amizades; na exposição de opiniões a colegas, professores e gestores; na defesa de concepções; e na aceitação ou negação de pedidos, percebendo as pistas fornecidas no comportamento do outro, como no exemplo do item 18 - "Posso enganar as pessoas sendo amigável".

Por outro lado, o fator internalizante (F2) propicia uma maior tolerância e sensibilidade às próprias necessidades e às do outro, como exemplificado no item 14 "Tenho problema em mudar meu comportamento para adequá-lo a diferentes pessoas e situações". Os fatores obtidos na análise dos resultados são corroborados por pesquisa de Guarino et al. (1998), descrita anteriormente, na qual os automonitores elevados apresentam comprometimento com as relações interpessoais na universidade e os automonitores com escore baixo apresentam maior interação acadêmica.

O presente estudo evidenciou a validade da Escala de Automonitoria para o contexto nacional, considerando ainda que não foi encontrado no país qualquer instrumento que permita mensurar a automonitoria. Como contribuição prática da versão traduzida da Escala de Automonitoria para o português do Brasil, tem-se a cooperação com pesquisas que investigam os diferentes aspectos da adaptação do estudante à universidade. A partir de tal perspectiva, seria producente identificar estudantes ingressantes que apresentam altos ou baixos escores em automonitoria, revelando aqueles alunos que apresentarão maior facilidade de engajamento acadêmico e/ou nas relações interpessoais, proporcionando-lhes ações mais pontuais que facilitem a integração à nova modalidade de ensino. Entretanto, apesar da pesquisa ter mostrado a confiabilidade da escala, os resultados obtidos não podem ser generalizados para um público mais amplo do que aquele formado por estudantes universitários, foco de todo este estudo. Além disso, alguns itens apresentaram valores pouco expressivos de correlação com os fatores encontrados. Nessa perspectiva, pesquisas futuras poderiam investigar a estrutura obtida para a escala e se esta permanece sem alterações significativas quando replicada em outra amostra.

\section{Agradecimentos}

Agradecimento em especial ao Professor Doutor Felipe Valentini pela revisão crítica do manuscrito.

\section{Referências}

Almeida, L. S., \& Castro, R. V. (2016). Ser estudante no ensino superior: Observatório dos percursos académicos dos estudantes da UMinho. Em L.S. Almeida \& R. V. Castro (Eds.), Ser estudante no Ensino Superior: O caso dos estudantes do1 ano (pp.15-31). Braga, Portugal: Universidade do Minho.

Amorim, R. (2001). A percepção da atractividade da fonte de comunicação em indivíduos com diferentes níveis de auto-monitoragem. Lisboa: Instituto Superior de Psicologia Aplicada.

Associação Brasileira de Empresas de Pesquisa (2015). Critério Brasil. Recuperado de http://www.abep.org

Avia, M. D., Sánchez-Bernardos, M. L., Sanz, J., Carrillo, J., \& Rojo, N. (1998). Self-presentation strategies and the five-factor model. Journal of Research in Personality, 32(1), 108-114. doi: 10.1006/jrpe.1997.2205

Bachner-Melman, R., Zohar, A. H., Kremer, I., Komer, M., Blank, S., Golan, M., \& Ebstein, R. P. (2009). Self-monitoring in anorexia nervosa. International Journal of Social Psychiatry, 55(2), 170-179. doi: 10.1177/0020764008094647

Bardagi, M. P., \& Hutz, C. S. (2012). Rotina acadêmica e relação com colegas e professores: impacto na evasão universitária. Psico, 43(2), 174-184.

Barreiros, J. (2011). Automonitoragem: processo baseado no comportamento prossocial. (Dissertação de Mestrado). Instituto de Universitário de Ciências Psicológicas, Sociais e da Vida-ISPA. Portugal: Lisboa.

Briggs, S. R., Cheek, J. M., \& Buss, H. A. (1980). An Analysis of the Self-Monitoring Scale. Journal of Personality and Social Psychology, 38(4), 679-686. doi: 10.1037/0022-3514.38.4.679

Briggs, S. R., \& Cheek, J. M (1988). On the Nature of Self-Monitoring: Problems with assessment, problems with validity. Journal of Personality and Social Psychology, 54(4), 663-678. doi: 10.1037/0022-3514.54.4.663

Fuglestad, P. T., \& Snyder, M. (2010). Status and the motivational foundations of self-monitoring. Social E Personality Psychology Compass, 4(11), 1031-1041. doi: 10.1111/j.1751-9004.2010.00311.x

Gangestad, S. W., \& Snyder, S. (2000). Self-Monitoring: Appraisal and Reappraisal. Psychological Bulletin, 126(4), 530-555. doi: 10.1037/00332909.126.4.530

Guarino, A, Michael, W. B., \& Hocevar, D. (1998). Self-Monitoring and student integration of community college students. The Journal of Social Psychology, 138(6), 754-757. doi: 10.1080/0022454980960326 
Ickes, W., Holloway, R., Stinson, L., \& Hoodenpyle, T. (2006). Self-Monitoring in social interaction: The centrality of self-affect. Journal of Personality, 74(3), 659-684. doi: 10.1111/j.1467-6494.2006.00388

Kodero, H. M. N. (1991). Self-monitoring: A cross-cultural study. (Unpublished thesis), University of Alberta, Canada.

Kyalo, P. M., \& Chumba, R. J. (2011). Selected factors influencing social and academic adjustment of undergraduate students of Egerton University; Njoro Campus. International Journal of Business and Social Science, 2(18), 274-290.

Lennox, R. D., \& Wolfe, R. N. (1984). Revision of the self-monitoring scale. Journal of Personality and Social Psychology, 46(6), $1349-1364$. doi: $10.1037 / 0022-3514.46 .6 .1349$

Lorenzo-Seva, U., \& Ferrando, P. J. (2006). Factor: A computer program to fit the exploratory factor analysis model. Behavior Research Methods, 38(1), 88-91. doi: 10.3758/BF03192753

Lorenzo-Seva, U., \& Van Ginkel, J. R. (2015). Multiple Imputation of missing values in exploratory factor analysis of multidimensional scales: estimating latent trait scores. Anales de Psicologia, 32(2), 506-608. doi: 10.6018/analesps.32.2.215161

Leone, C. (2006). Self-monitoring: Individual differences in orientations to the social world. Journal of Personality, 74(3), 633-657. doi: 10.1111/j.1467-6494.2006.00387.x

Mislevy, R. J., \& Bock, R. D. (1990). Bilog 3 Item analysis and test scoring with binary logistic models. Mooresville: Scientific Software.

Neto, F. (1993). Love styles and self-presentations. Personality and Individual Differences, 14(6), 795-803.

Nowack W., \& Kammer, D. (1987). Self-presentation: Social skills and inconsistency as independent facets of self-monitoring. European Journal of Personality, 1(2), 61-77. doi: 10.1002/per.2410010202

Olsson, U. (1979a). Maximum likelihood estimation of the polychoric correlation coefficient. Psychometrika, 44(4), 443-460. doi: 10.1007/ BF02296207

Olsson, U. (1979b). On the robustness of factor analysis against crude classification of the observations. Multivariate Behavioral Research, 14(4), 485-500. doi: 10.1207/s15327906mbr1404_7

Oyamot, C. M., Fuglestad, P. T., \& Snyder, M. (2010). Balance of power and influence in relationships: The role of self-monitoring. Journal of Social and Personal Relationships, 27(1), 23-46. doi: 10.1177/0265407509347302

Pérez, P. R. A., Santiviago, C, Aguilar, D. L., DaRe, L., \& Rubio, V. (2014). Competencias de adaptabilidad y expectativas del alumnado en proceso de transición a la educación superior: Un estudio transnacional en España, Uruguay e Italia. Actas Cuarta Conferencia Latinoamericana sobre el abandono em la Educación Superior, 1-10.

Polanco, A., Ortiz, L., Pérez, C., Parra, P., Fasce, E., Matus, O., ... \& Meyer, A. (2014). Relación de antecedentes académicos y expectativas iniciales con el bienestar académico de alumnos de primer año de medicina. Fundacione de Educacione Medica, 17(4), 205-211. doi: 10.4321/S2014-98322014000400006

Sasovova, Z. (2006). To dislike and to be liked: Self-monitoring, affect-intensive relations and work performance. Academy of management proceedings, 1, 1-6. doi: 10.5465/AMBPP.2006.22898634.

Smidt, K. E., \& DeBono, K. G. (2011). On the effects of product name on product evaluation: An individual difference perspective. Social influence, 6(3), 131-141. doi: 10.1080/15534510.2011.578501

Snyder, M. (1974). Self-monitoring of expressive behavior. Journal of Personality and Social Psychology, 30(4), 526-537. doi: 10.1037/h0037039

Snyder, M., \& Gangestad, S. (1986). On the Nature of Self-Monitoring: Matters of Assessment, Matters of Validity. Journal of Personality and Social Psychology, 51(1), 125-139. doi: 10.1037/0022-3514.51.1.125

Snyder, M. (1987). Public appearances/private realities: The psychology of self-monitoring. New York: Freeman.

Soares, A. B, Poubel, L. N., \& Melo, T. V. S. (2009). Habilidades sociais e adaptação acadêmica: um estudo comparativo em instituições de ensino público e privado. Aletheia, $s / v(29), 27-42$.

Soibel, A., Fong, K., Mullin, J.B., Jenkins, G., \& Mar, R. A. (2012). Is self-monitoring related to social comparison? It depends how you ask. Individual Differences Research, 10(4), 193-201.

Teixeira, M. A. P., Dias, A. C. G., Wottrich, S. H., \& Oliveira, A. M. (2008). Adaptação à universidade em jovens calouros. Psicologia Escolar e Educacional, 12(1), 185-202. doi: 10.1590/S1413-85572008000100013

Ten Berge, J. M. F., \& Kiers, H. A. L. (1991). A numerical approach to the exact and the approximate minimum rank of a covariance matrix. Psychometrika, 56(2), 309-315. doi: 10.1007/BF02294464

Timmerman, M. E., \& Lorenzo-Seva, U. (2011). Dimensionality assessment of ordered polytomous items with parallel analysis. Psychological Methods, 16(2), 209-220. doi: 10.1037/a0023353

Wilmot, M. P. (2015). A contemporary taxometric analysis of the latent structure of self-monitoring. Psychological Assessment, 27(2), 353-364. doi: 10.1037/pas0000030.supp

Wilmot, M. P., DeYoung, C. G., Stillwell, D., \& Kosinski, M. (2015). Self-monitoring and the metatraits, Journal of Personality, 84(3), 335-347. doi: 10.1111/jopy.12162

\section{Sobre as autoras}

Marcia Cristina Monteiro é Psicóloga, Doutoranda em Psicologia Social pela Universidade Salgado de Oliveira (UNIVERSO) e Professora da União das Instituições de Ensino de São Paulo (UNIESP) na Faculdade Duque de Caxias.

Adriana Benevides Soares é Psicóloga, Doutora pela Universidade de Paris XI, Pós-doutora pela Universidade Federal de São Carlos (UFSCar), Professora da Universidade Salgado de Oliveira (UNIVERSO) e Professora da Universidade do Estado do Rio de Janeiro (UERJ). 